\title{
Near field diffraction of cylindrical convex gratings
}

\author{
Francisco Jose Torcal-Milla, Luis Miguel Sanchez-Brea and \\ Eusebio Bernabeu
}

Applied Optics Complutense Group, Optics Department, Universidad Complutense de Madrid, Facultad de Ciencias Físicas, Ciudad Universitaria s.n., 28040, Madrid, Spain

E-mail: fjtorcal@ucm.es

Received 7 October 2014, revised 12 December 2014

Accepted for publication 31 December 2014

Published DD MM 2014

\begin{abstract}
We analyze the field produced by a cylindrical convex diffraction grating at the Fresnel regime for several kinds of light sources, including a monochromatic quasipunctual source, finite size, and polychromatic sources. These results can help one understand the functioning of rotary optical encoder technology. A decrease in the self-image contrast is produced for finite nonpunctual sources. In addition, the polychromaticity of the source affects the smoothness of the self-images, making them quasicontinuous from a certain distance from the grating forward. Finally, we experimentally validate the obtained analytical predictions.
\end{abstract}

Keywords: diffraction gratings, self-imaging, polychromatic light

PACS numbers: 42.25.Fx, 42.79.Dj

SQ1 (Some figures may appear in colour only in the online journal)

\section{Introduction}

Diffraction gratings are optical elements that periodically modulate one or more properties of light passing through them or reflecting from them $[1,2]$. The most common diffraction gratings are those that modulate the amplitude or the phase of the incident field [2]. The applicability of diffraction gratings is quite extensive. One can find them as fundamental parts of many different kinds of devices, such as telescopes, spectrometers, and optical encoders [1, 3-5]. For example, the self-imaging phenomenon produced at the near field $[3,6]$ is used in diffraction-based optical encoders to measure the relative position and/or displacement between two different parts. Due to their robustness and precision, optical encoders are used in machine tools, robotics, motion control, and more. They are formed by an illumination source, a diffraction grating - commonly called scale-a mask, and some photodetectors. The relative displacement between the scale and the reading head produces a variation in the photodetector's signals and gives the measurement of the linear (linear encoders) or angular (rotary encoders) movement. In particular, rotary encoders use a diffraction grating engraved on a cylindrical substrate. Usually, gratings for rotary optical encoders are engraved by lithographic methods on the plane facet of a cylinder [7, 8]. The behavior of this kind of grating has been already analyzed in previous works $[9,10]$. On the other hand, rotary encoders with the grating engraved by laser ablation [11, 12] on the external curved side of a steel cylinder have recently been developed [13-15]. It has been demonstrated that gratings engraved by nanosecond laser ablation on steel substrates act like amplitude gratings due to their surficial roughness [16, 17].

For plane diffraction gratings, a deep analysis of the nearfield and far-field diffraction has been performed in recent decades, including the near-field pattern when the grating is illuminated with polychromatic and finite extension sources $[18,19]$. The diffraction pattern and image formation produced by concave curved gratings-such as torus, spherical, etc-has also been analyzed in several works [20-23]. Since curved gratings have generated interest in recent years, a more complete analysis needs to be performed to optimize the behavior of optical encoders and other near-field applications. For example, the near-field diffraction of convex gratings engraved on the external side of a cylinder and illuminated by a monochromatic plane wave has recently been analyzed [24].

In the present work, we extend the research to curved, convex diffraction gratings illuminated by a punctual or finite source that also may be monochromatic or polychromatic. We 


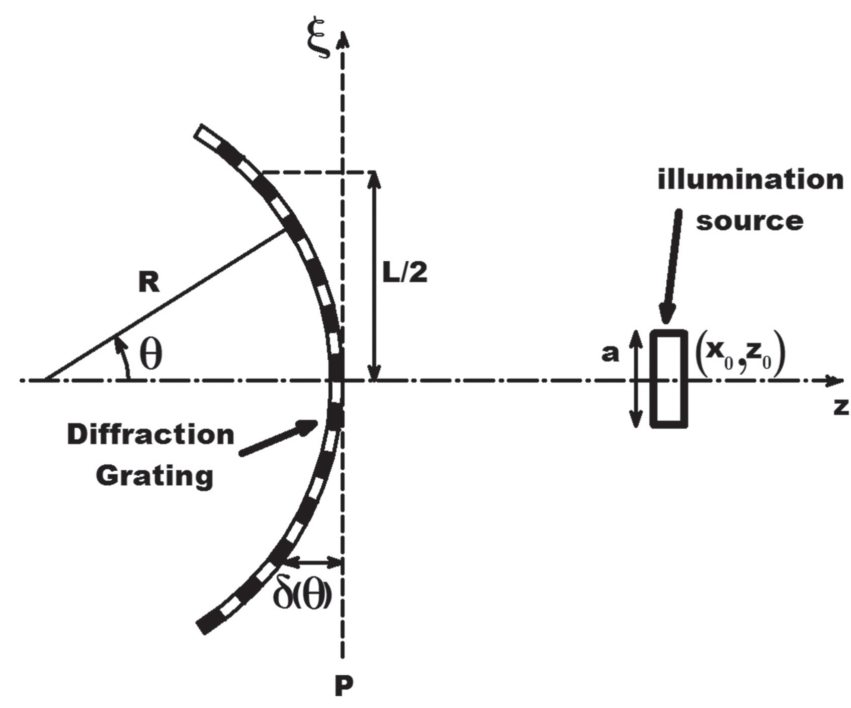

Figure 1. Scheme of the analyzed configuration, where $(\xi, z)$ are the coordinates centered at the point where the grating crosses the $z$-axis, $\mathrm{P}$ is a plane perpendicular to the optical axis ( $z$-axis) at the origin of coordinates, $\delta(\theta)$ is the distance between each point of the grating and the plane $\mathrm{P}, R$ is the curvature radius of the grating, $L$ is the area of the grating illuminated by the source over the $\xi$-axis, and $a$ is the size of the source placed at the point $\left(x_{0}, z_{0}\right)$. The black and white regions of the grating represent nonreflective and reflective areas, respectively.

present analytical equations for the self-imaging process for the considered cases, and we experimentally verify the most general case. The obtained results are directly applicable to rotary optical encoders.

\section{Theoretical approach}

To analyze the general case of a polychromatic and finite extension source, we first consider the simple case of a monochromatic and punctual source placed at a distance, $z_{0}$, from a cylindrical convex diffraction grating and at a distance, $x_{0}$, from the $z$-axis. The scheme of the setup is shown in figure 1. Light propagates from right to left until it impinges the diffraction grating and is diffracted back. In this figure, $P$ is a plane perpendicular to the optical axis (z-axis) at the origin of coordinates, $\delta(\theta)$ is the distance between each point of the grating and the plane $\mathrm{P}, R$ is the curvature radius of the grating, $(\xi, z)$ are the coordinates centered at the point where the grating crosses the $z$-axis, $a$ is the size of the source, and $L$ is the area of the grating illuminated by the source over the $\xi$ axis. The radius of curvature is defined to be positive when the center of the curvature is placed at the left side of the grating. In addition, black and white regions of the grating represent nonreflective and reflective areas, respectively.
In a parabolic approximation, the field produced by a punctual source at a distance, $z_{0}$, is given by

$$
U_{0}=A_{0} \frac{\exp \left(i k z_{0}\right)}{\sqrt{z_{0}}} \exp \left[\frac{i k\left(\xi-x_{0}\right)^{2}}{2 z_{0}}\right],
$$

where $A_{0}$ is the amplitude, $x_{0}$ is the position of the source along the $x$-axis, $k=2 \pi / \lambda$, and $\lambda$ is the illumination wavelength. To calculate the diffraction pattern produced by the grating, we consider the three-dimensional topography by computing the optical path. Each point of the wavefront along the $\xi$-axis covers a different distance from the plane, $\mathrm{P}$, to the surface of the grating. The optical path for each point of the wavefront at $\xi(\theta)=R \sin \theta$ is $\delta(\theta)=R(1-\cos \theta)$. Then, each point is affected by the propagation coefficient, $t(\theta)=\exp [i k R(1-\cos \theta)]$, which can be expressed in Cartesian coordinates as

$$
t(\xi)=\exp \left\{i k R\left[1-\sqrt{1-\left(\frac{\xi}{R}\right)^{2}}\right]\right\}
$$

where $t(\xi)$ represents the propagation coefficient due to the optical path of each point of the wavefront from the plane, $\mathrm{P}$, to the surface of the grating. The diffraction grating is angularly periodic, not linearly periodic. Then, its reflection coefficient can be expressed as a Fourier series expansion depending on the angle, $\theta$,

$$
r(\theta)=\sum_{n} a_{n} \exp \left(i q_{\theta} n \theta\right),
$$

where $a_{n}$ are the Fourier coefficients with $n$ integer, $q_{\theta}=2 \pi / p_{\theta}$, and $p_{\theta}$ is the angular period whose relationship with the linear period, $p$, along the perimeter is $p_{\theta}=p / R$. Changing to Cartesian coordinates, equation (3) results in

$$
r(\xi)=\sum_{n} a_{n} \exp \left[i q_{\theta} n \arcsin \left(\frac{\xi}{R}\right)\right] .
$$

This corresponds to the Fourier series expansion of the reflectance produced by the grating expressed in Cartesian coordinates. From equations (1), (2), and (4), the field at the plane, $\mathrm{P}$, after being diffracted by the grating is

$$
\begin{aligned}
U_{1}(\xi, z=0)= & U_{0}(\xi, z=0) t(\xi) r(\xi) t(\xi) \\
& \propto A_{0} \frac{\exp \left[i k z_{0}\right]}{\sqrt{z_{0}}} \exp \left[\frac{i k\left(\xi-x_{0}\right)^{2}}{2 z_{0}}\right] \\
& \times \exp \left\{2 i k R\left[1-\sqrt{1-\left(\frac{\xi}{R}\right)^{2}}\right]\right\} \\
& \times \sum_{n} a_{n} \exp \left[i q_{\theta} n \arcsin \left(\frac{\xi}{R}\right)\right] .
\end{aligned}
$$

The field is obtained by the product of the incident field, the reflectance of the grating, and the phase difference corresponding to each point of the wavefront under thin element approximation. Since light covers twice the distance from the 
plane, $\mathrm{P}$, to the grating due to the reflective configuration, the optical path, $\delta(\theta)$, doubles its value.

To calculate the field propagation from the plane, $\mathrm{P}$, forward, we use the Fresnel approach $(p \gg \lambda)$,

$$
\begin{aligned}
& U_{2}(x, z)=\frac{\exp (i k z)}{\sqrt{i k z}} \int_{-L / 2}^{L / 2} U_{1}(\xi, z=0) \\
& \times \exp \left[\frac{i k(x-\xi)^{2}}{2 z}\right] d \xi
\end{aligned}
$$

where $x$ is the coordinate perpendicular to the propagation axis at the observation plane. To solve equation (6), we consider that the radius is much longer than the illuminated area of the grating, $R \gg L$. Then, we can perform a Taylor series expansion in the exponents of equation (5). In addition, we extended the integral limits of equation (6) to infinity since areas of the grating far from the optical axis do not contribute to the field around the optical axis at near distances. Solving the integral, the intensity calculated as $I_{2}(x, z)=U_{2}(x, z) U_{2}^{*}(x, z)$ results in

$$
\begin{aligned}
& I_{2, M}^{P S}(x, z) \propto \frac{\left|A_{0}\right|^{2} R}{R\left(z+z_{0}\right)+2 z z_{0}} \sum_{n, n^{\prime}} a_{n} a_{n^{\prime}}^{*} \\
& \quad \times \exp \left[\frac{i q_{\theta}\left(n-n^{\prime}\right)\left(z x_{0}+x z_{0}\right)}{R\left(z+z_{0}\right)+2 z z_{0}}\right] \\
& \quad \times \exp \left\{-\frac{i q_{\theta}^{2}\left(n^{2}-n^{\prime 2}\right) z z_{0}}{2 k R\left[R\left(z+z_{0}\right)+2 z z_{0}\right]}\right\},
\end{aligned}
$$

where $*$ denotes complex-conjugate, $n$ and $n^{\prime}$ are integers, the superindex $P S$ denotes punctual source, and the subindex, $M$, indicates monochromatic. Both effects-the curvature of the grating and the spherical wave coming from the punctual source-are present in the intensity. The effects are basically the same, affecting the period of the fringes and also the distance between the self-images along the $z$-axis. In figure 2, we show two examples of the diffracted intensity considering a Ronchi convex diffraction grating [1, 2]. We observed how divergent and convergent incoming light affects the diffracted intensity at the near field. The period of the self-images and the distance between them vary. From equation (7), we can extract the expressions for the period of the self-images and the period of the self-imaging phenomenon along the $z$-axis. From the first exponential factor of equation (7), the period of the self-images, $\hat{p}$, depends on the radius and the distance between the source and the grating as follows

$$
\hat{p}=p\left[1+z\left(\frac{1}{z_{0}}+\frac{2}{R}\right)\right] \text {. }
$$

The period increases linearly in terms of $z$ for fixed values of $z_{0}$ and $R$. On the other hand, the period of the selfimages phenomenon along the $z$-axis also depends on the
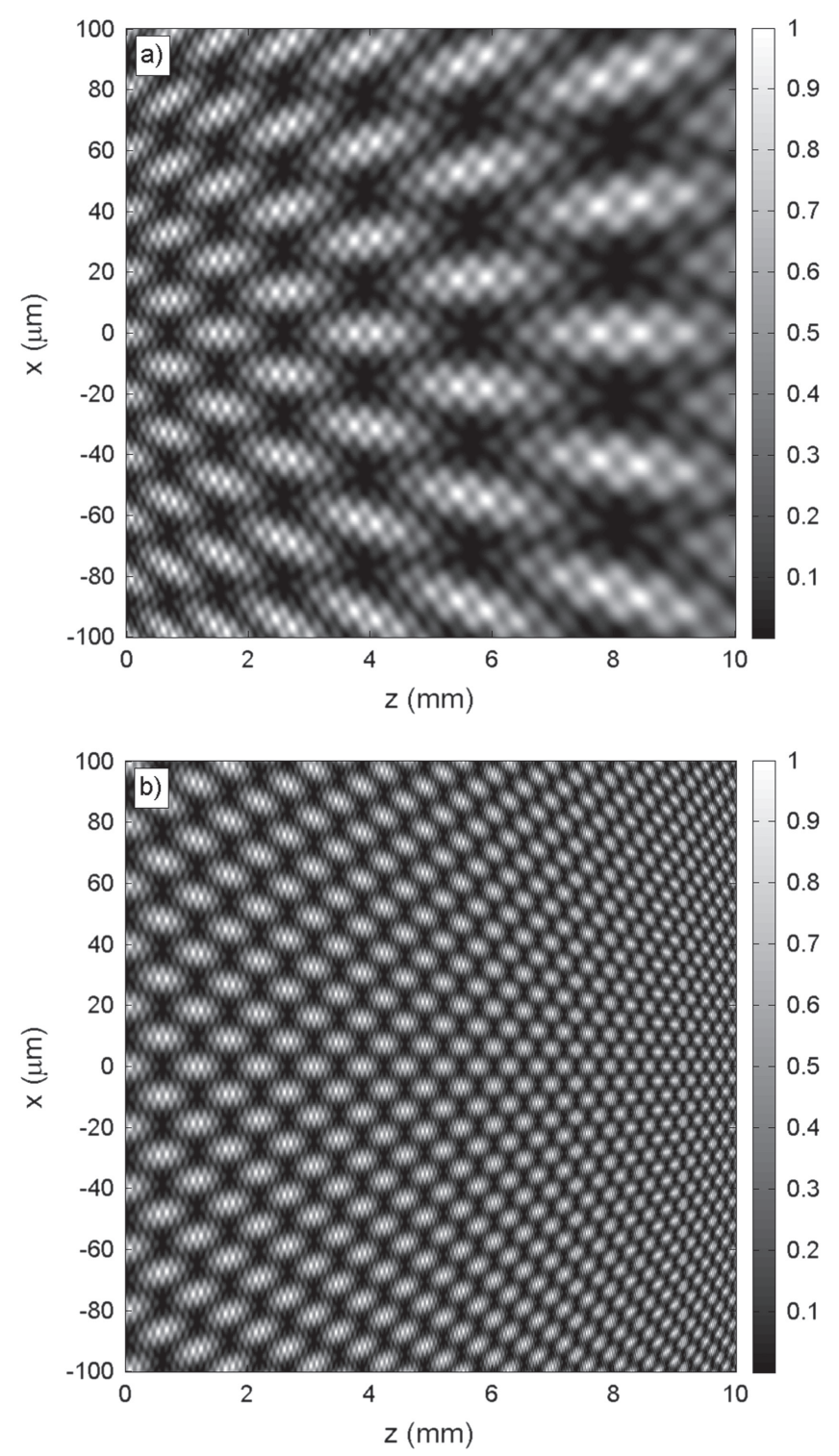

Figure 2. Analytical self-images for a Ronchi cylindrical convex diffraction grating with period $p=20 \mu \mathrm{m}, R=50 \mathrm{~mm}$, illumination wavelength $\lambda=0.6328 \mu \mathrm{m}$, and taking the first three diffraction orders. The punctual monochromatic source is placed at $x_{0}=0 \mu \mathrm{m}$, (a) $z_{0}=10 \mathrm{~mm}$, (b) $z_{0}=-10 \mathrm{~mm}$.

radius of the grating and the source distance as

$$
\hat{z}_{T}=z_{T}\left[1+z\left(\frac{1}{z_{0}}+\frac{2}{R}\right)\right]^{2}
$$

It responds to a quadratic form for fixed values of $z_{0}$ and $R$. The parameter, $z_{T}=2 p^{2} / \lambda$, is the Talbot distance for a linear diffraction grating with period, $p$, illuminated by a plane wave [3].

An interesting example occurs when the light source is placed on an axis, $x_{0}=0$, at a distance of $z_{0}=-R / 2$ from the grating, which corresponds to a virtual convergent light source. Then, the diffracted intensity exactly corresponds with what would be produced by a plane wave impinging on a 
plane diffraction grating,

$$
\begin{aligned}
& I_{2, M}^{P W}(x, z) \propto\left|A_{0}\right|^{2} \sum_{n, n^{\prime}} a_{n} a_{n^{\prime}}^{*} \exp \left[\frac{i 2 \pi\left(n-n^{\prime}\right) x}{p}\right] \\
& \quad \times \exp \left\{-\frac{i 2 \pi\left(n^{2}-n^{\prime 2}\right) z}{z_{T}}\right\},
\end{aligned}
$$

where we have used the relationships, $q_{\theta}=q R, q=2 \pi / p$, and superindex $P W$ denotes the plane wave output.

Up to this point, we have considered an ideal punctual illumination source. Nevertheless, standard illumination sources such as LEDs are neither punctual nor monochromatic, but they have finite extension and a certain emission spectrum. Therefore, we will compute the intensity distribution diffracted by the considered grating for a polychromatic and finite-size source. To do this, we simply need to integrate equation (7) over $\lambda$ considering the spectrum $g(\lambda)$, and over $x_{0}$ considering that the size of the source is $a$

$$
I_{2, P}^{F S}(x, z) \propto \int_{-a / 2}^{a / 2} \int g(\lambda) I_{2, M}^{P S}\left(x, z, x_{0}, \lambda\right) \mathrm{d} \lambda \mathrm{d} x_{0} .
$$

To obtain analytical results, we particularize to an uniform spectrum, centered at $\lambda_{0}$ with a width of $\Delta \lambda$. We also consider that all the points of the source emit in the same way. Then, the wavelength integral runs from $\lambda_{0}-\Delta \lambda / 2$ to $\lambda_{0}+\Delta \lambda / 2$, and the size of the source integral in $x_{0}$ runs from $-a / 2$ to $a / 2$. The intensity results in

$$
\begin{aligned}
I_{2, P}^{F S}(x, z)= & \int_{-a / 2}^{a / 2} \int_{\lambda_{0}-\Delta \lambda / 2}^{\lambda_{0}+\Delta \lambda / 2} I_{2, M}^{P S}\left(x, z, x_{0}, \lambda\right) \mathrm{d} \lambda \mathrm{d} x_{0} \\
= & \left|A_{0}\right|^{2} \sum_{n, n^{\prime}} a_{n} a_{n^{\prime}}^{*} \exp \left[\frac{i q_{\theta}\left(n-n^{\prime}\right) x z_{0}}{R\left(z+z_{0}\right)+2 z z_{0}}\right] \\
& \times \exp \left\{-\frac{i q_{\theta}^{2}\left(n^{2}-n^{\prime 2}\right) z z_{0}}{2 k R\left[R\left(z+z_{0}\right)+2 z z_{0}\right]}\right\} \\
& \times \operatorname{sinc}\left\{\frac{\left(n^{2}-n^{\prime 2}\right) q_{\theta}^{2} z z_{0} \Delta \lambda}{8 \pi R\left[R z+(R+2 z) z_{0}\right]}\right\} \\
& \times \operatorname{sinc}\left\{\frac{a\left(n-n^{\prime}\right) q_{\theta} z}{2\left[R z+(R+2 z) z_{0}\right]}\right\},
\end{aligned}
$$

where $\operatorname{sinc} \alpha=\sin \alpha / \alpha, F S$ means finite source, and $P$ means polychromatic source. As one can see, the intensity is affected by two sinc functions due to the polychromaticity and finite size of the source, respectively. All the performed analyses are still valid for lower periods, provided that $p \gg \lambda$ and diffractive effects occur.

To continue, let us compare the effects of both sinc functions by means of their widths, $\omega_{\Delta \lambda}, \omega_{a}$, defined as the value, $z$, that fulfills $\operatorname{sinc} \alpha=0$ for each $\operatorname{sinc}$ function,

$$
\left\{\begin{array}{l}
\omega_{\Delta \lambda}=\frac{z_{0}}{\left(n^{2}-n^{\prime 2}\right) z_{0} \Delta \lambda / 2 p^{2}-\left(1+2 z_{0} / R\right)}, \\
\omega_{a}=\frac{z_{0}}{\left(n-n^{\prime}\right) a / p-\left(1+2 z_{0} / R\right)} .
\end{array}\right.
$$

We may compare both widths by dividing them,

$$
\frac{\omega_{\Delta \lambda}}{\omega_{a}}=\frac{\left(n-n^{\prime}\right) a / p-\left(1+2 z_{0} / R\right)}{\left(n^{2}-n^{\prime 2}\right) z_{0} \Delta \lambda / 2 p^{2}-\left(1+2 z_{0} / R\right)} .
$$

The sinc function with the lesser width will be predominant. The polychromaticity of the source is predominant when $\omega_{\Delta \lambda}<\omega_{a}$, resulting in an achromatic self-imaging regime from a certain distance forward; the finite size of the source is predominant for $\omega_{\Delta \lambda}>\omega_{a}$, attenuating the fringes for long distances from the grating. This result is of interest in rotary optical encoder technology, where the self-imaging phenomenon is an important issue to solve.

In figures 3(a) and (b), we show two examples of the selfimages produced by a convex cylindrical Ronchi grating illuminated by a polychromatic and finite extension source. These examples correspond to the same parameters as figure 2 , but they take into consideration polychromatic light with $\Delta \lambda=50 \mathrm{~nm}$ and lateral size $a=50 \mu \mathrm{m}$. In this particular example, $\omega_{\Delta \lambda}>\omega_{a}$ and the contrast of the fringes decay to zero from a certain distance forward. This effect is better observed in figure 3(b). These results show that diffraction fringes only occur up to a certain distance. Therefore, this configuration may be used in optical encoders, provided that the reading head is placed closer to the grating than the mentioned distance. In addition, in figures 3(c) and (d) we show another example in which the term, $\omega_{\Delta \lambda}<\omega_{a}$, is predominant due to polychromaticity. It corresponds to the same parameters as figure 2 , but takes into consideration polychromatic light with $\Delta \lambda=50 \mathrm{~nm}$ and lateral size $a=10 \mu \mathrm{m}$.

For completeness, other particular cases corresponding to finite monochromatic sources and punctual polychromatic sources are analyzed and shown in the appendix. They are obtained from equation (12). These cases are of interest in other configurations of optical rotary encoders.

\section{Experimental approach}

To corroborate the theoretical results obtained in the previous section, we carried out the experiment shown in figure 4 . We used a diffraction grating of period $p=20 \mu \mathrm{m}$ engraved by laser ablation on a cylindrical steel substrate. The nature of the substrate allows it to bend into a cylindrical shape. In our practical case, we engraved a grating approximately two centimeters long, which is enough to validate the analytical results. The radius of curvature of the grating was $R=50 \mathrm{~mm}$. We used an LED of wavelength $\lambda=655 \pm 20 \mathrm{~nm}$ as the illumination source. The emitting area was circular, with the diameter $a=57 \mu \mathrm{m}$. Afterward, light was captured by a CMOS camera ( $\mu$ Eye by IDS, pixel size 

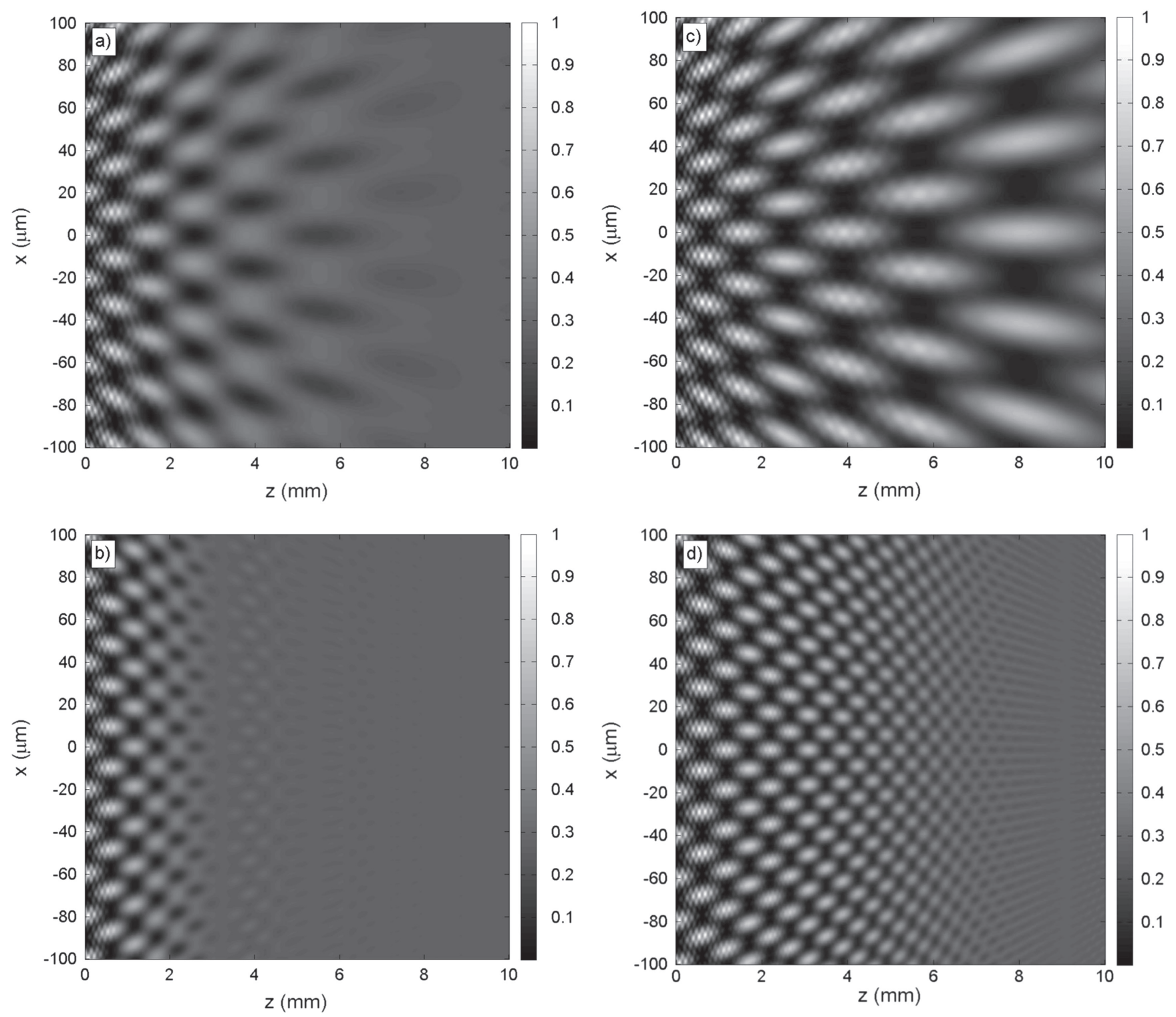

Figure 3. Analytical self-images for a Ronchi cylindrical convex diffraction grating with period $p=20 \mu \mathrm{m}, R=50 \mathrm{~mm}$, polychromatic illumination $\lambda=0.6328 \pm 0.025 \mu \mathrm{m}$, and taking the first three diffraction orders, the finite source of size $a$ placed at $x_{0}=0 \mu \mathrm{m}$. (a) $a=50 \mu \mathrm{m}, z_{0}=10 \mathrm{~mm}$, (b) $a=50 \mu \mathrm{m}, z_{0}=-10 \mathrm{~mm}$, (c) $a=10 \mu \mathrm{m}, z_{0}=10 \mathrm{~mm}$, (d) $a=10 \mu \mathrm{m}, z_{0}=-10 \mathrm{~mm}$.

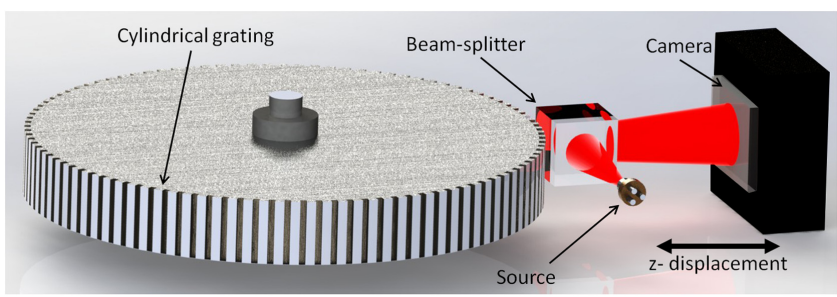

Figure 4. Scheme of the experimental setup. In our practical case, we have engraved a grating approximately two centimeters long, which is enough to validate the analytical results.

$2.2 \times 2.2 \mu \mathrm{m}$ ) coupled to a microscope objective, which was used to magnify the self-images. We used a microscope objective (model: M Plan Apo NIR 50× by Mitutoyo) to capture images from the grating surface backward through the beam splitter. In addition, the camera was free to travel along the propagation axis. The experiment consisted of separating the camera from the grating plane along the propagation axis and acquiring images of the diffracted intensity at different planes. Figure 5 shows that the obtained analytical (figure 5(a)) - from equation (12)—and experimental (figure 5(b)) results for this particular case have good agreement.

\section{Conclusions}

In this work, we analyzed the near-field intensity pattern of cylindrical convex diffraction gratings illuminated by a general source that can be punctual or finite, monochromatic or polychromatic. We analyzed the effects of the source size and 

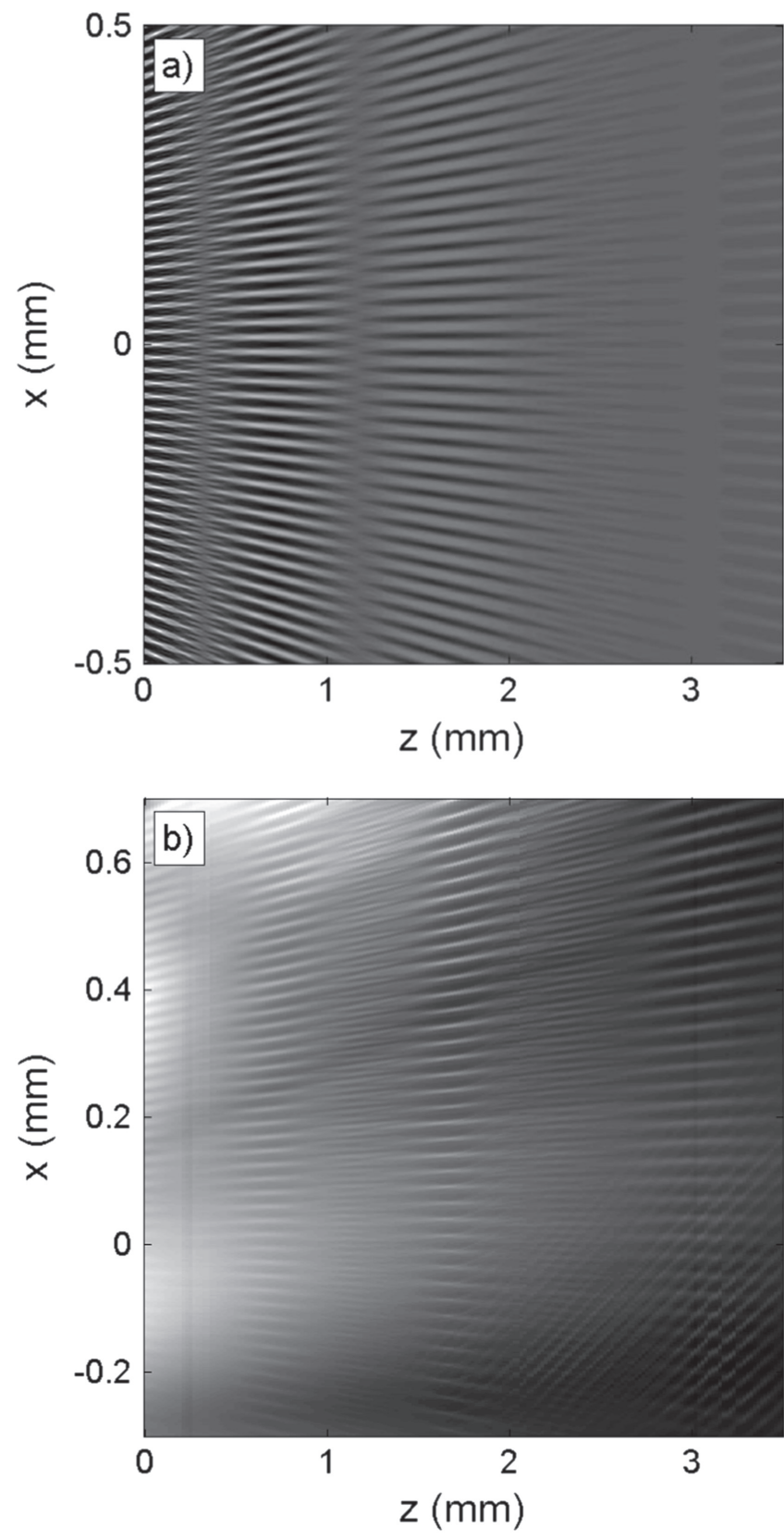

Figure 5. Self-images for a Ronchi cylindrical convex diffraction grating with period $p=20 \mu \mathrm{m}, R=50 \mathrm{~mm}$, polychromatic illumination $\lambda=0.655 \pm 0.02 \mu \mathrm{m}$, finite source with width $a=57 \mu \mathrm{m}$ placed at $x_{0}=0 \mu \mathrm{m}$ and $z_{0}=5 \mathrm{~mm}$. (a) Analytical, taking the first three diffraction orders. (b) Experimental.

polychromaticity of the source, giving analytical expressions for all cases. In addition, we performed experiments to corroborate the most general analytical result: the finite polychromatic source. These analyses and results can help one understand the behavior of optical rotary encoder behavior with this kind of grating.
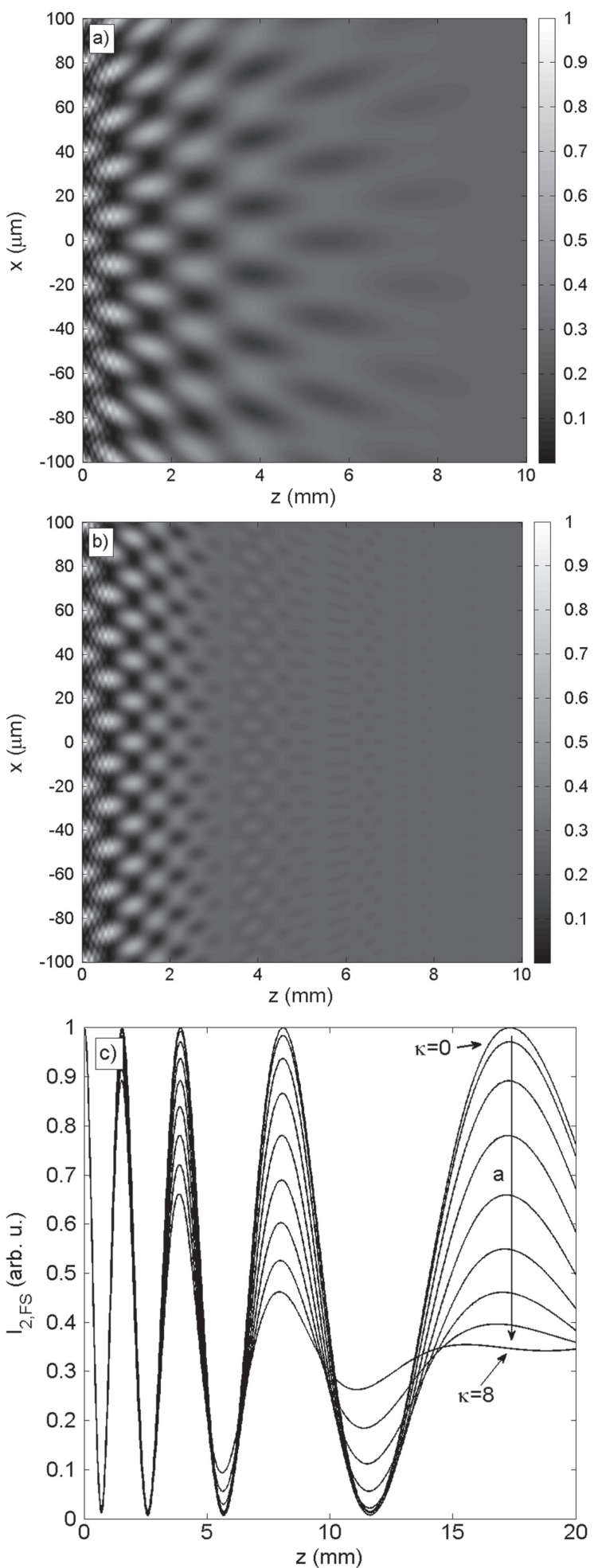

Figure 6. Analytical self-images for a Ronchi cylindrical convex diffraction grating with period $p=20 \mu \mathrm{m}, R=50 \mathrm{~mm}$, illumination wavelength $\lambda=0.6328 \mu \mathrm{m}$, and taking the first three diffraction orders, finite source of size $a=50 \mu \mathrm{m}$ placed at $x_{0}=0 \mu \mathrm{m}$. (a) $z_{0}=10 \mathrm{~mm}$, (b) $z_{0}=-10 \mathrm{~mm}$, (c) intensity along the optical axis for different sizes of the source $a=\kappa \times 5 \mu \mathrm{m}(\kappa=0, \ldots, 8)$ 

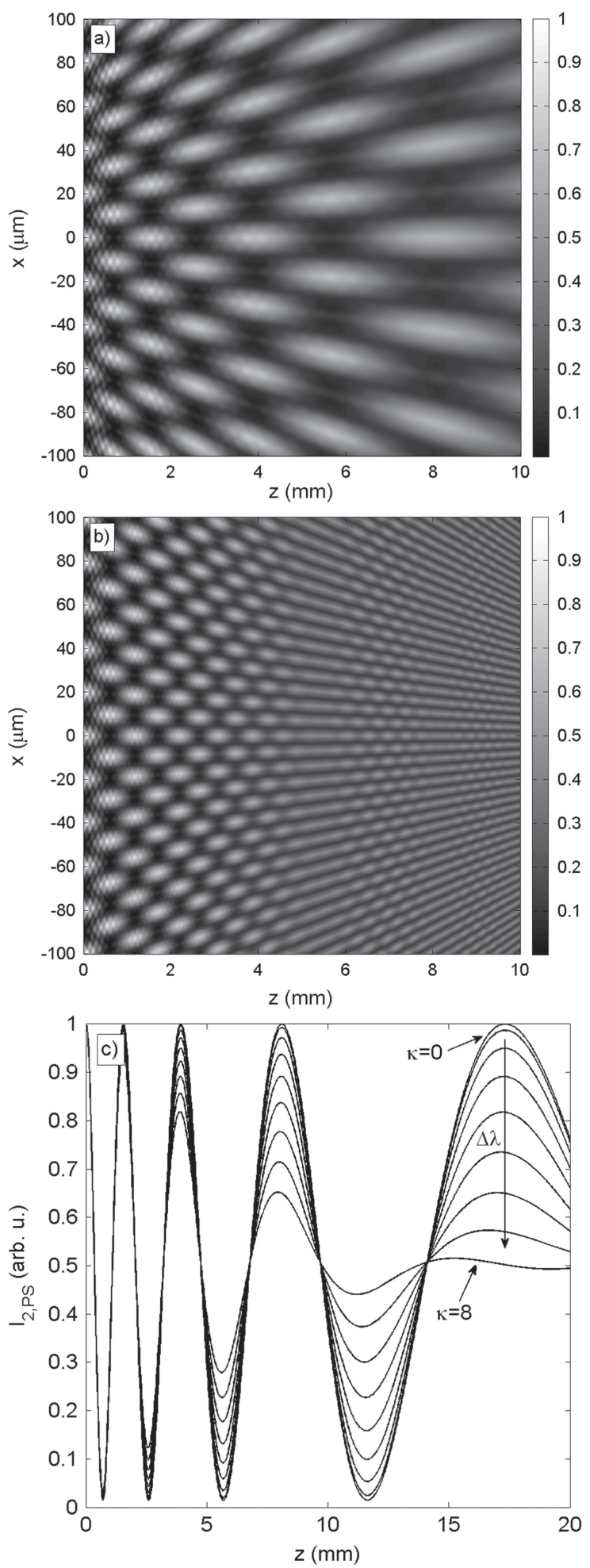

Figure 7. Analytical self-images for a Ronchi cylindrical convex diffraction grating with period $p=20 \mu \mathrm{m}, R=50 \mathrm{~mm}$, polychromatic illumination $\lambda=0.6328 \pm 0.05 \mu \mathrm{m}$, and taking the first three diffraction orders, punctual source placed at $x_{0}=0 \mu \mathrm{m}$. (a) $z_{0}=10 \mathrm{~mm}$, (b) $z_{0}=-10 \mathrm{~mm}$, (c) intensity along the optical axis for different spectrum widths, $\Delta \lambda=\kappa \times 0.2 \mu \mathrm{m}(\kappa=0, \ldots, 8)$.

\section{Acknowledgments}

The authors thank Jose Maria Herrera-Fernandez for his help with graphics. This work has been supported by the project DPI2011-27851 of the Ministry of Science and Innovation of Spain and the project Fagor Automation-UCM Ref. 92/2014.

\section{Appendix}

In this appendix, we show the two intermediate cases- the finite monochromatic source and punctual polychromatic source-by particularizing equation (12).

\section{Finite monochromatic source}

We obtain the intensity distribution for a monochromatic finite light source of lateral size, $a$, by particularizing equation (12) for $\Delta \lambda=0$. It results in

$$
\begin{aligned}
& I_{2, M}^{F S}(x, z) \propto\left|A_{0}\right|^{2} \sum_{n, n^{\prime}} a_{n} a_{n^{\prime}}^{*} \\
& \times \exp \left\{-\frac{i q_{\theta}^{2}\left(n^{2}-n^{\prime 2}\right) z z_{0}}{2 k R\left[R\left(z+z_{0}\right)+2 z z_{0}\right]}\right\} \\
& \times \exp \left[\frac{i q_{\theta}\left(n-n^{\prime}\right) x z_{0}}{R\left(z+z_{0}\right)+2 z z_{0}}\right] \\
& \quad \times \operatorname{sinc}\left\{\frac{a\left(n-n^{\prime}\right) q_{\theta} z}{2\left[R z+(R+2 z) z_{0}\right]}\right\},
\end{aligned}
$$

where the superindex FS denotes finite source, and we have considered the source centered at the optical axis, $\left(x_{0}=0\right)$. The effect of considering a finite source is a loss of intensity and contrast when we separate from the grating. In figures 6(a) and (b), we show two examples with the same parameters as figures 2 (a) and (b), but considering $\Delta \lambda=0$. The effect of the sinc function is better observed in figure 6(b), presenting areas with high and low contrast along the $z$-axis. In this case, the periods of the self-images along the $x$-axis and the $z$-axis are also affected in the same fashion as in equations (8) and (9).

In addition, figure 6(c) shows the intensity dependence along the optical axis in terms of the size of the source for the finite monochromatic source. In this case, the fringes disappear from a certain distance forward, and the intensity stabilizes at a certain constant value. This occurs because the intensity along the optical axis tends to a constant value.

\section{Punctual polychromatic source}

We obtain the diffracted intensity produced by a polychromatic punctual source placed at a distance, $z_{0}$, by 
particularizing equation (12) for $a=0$. It results in

$$
\begin{aligned}
& I_{2, P}^{P S}(x, z) \propto\left|A_{0}\right|^{2} \sum_{n, n^{\prime}} a_{n} a_{n^{\prime}}^{*} \exp \left[\frac{i q_{\theta}\left(n-n^{\prime}\right)\left(z x_{0}+x z_{0}\right)}{R\left(z+z_{0}\right)+2 z z_{0}}\right] \\
& \quad \times \exp \left\{-\frac{i q_{\theta}^{2}\left(n^{2}-n^{\prime 2}\right) z z_{0}}{2 k R\left[R\left(z+z_{0}\right)+2 z z_{0}\right]}\right\} \\
& \quad \times \operatorname{sinc}\left\{\frac{\left(n^{2}-n^{\prime 2}\right) q_{\theta}^{2} z z_{0} \Delta \lambda}{8 \pi R\left[R z+(R+2 z) z_{0}\right]}\right\},
\end{aligned}
$$

where the subindex $P$ indicates polychromatic, and we have considered the source centered at the optical axis, $\left(x_{0}=0\right)$. In figures 7(a) and (b), we show two examples of intensity diffracted by a curved grating illuminated by a polychromatic punctual source. These examples correspond to the same parameters as those in figures 2(a) and (b), but considering $a=0$. The effect is similar to that obtained for a finite monochromatic source. A decay of the intensity is produced for long distances from the grating, but the polychromaticity of the beam has an additional effect. From a certain distance forward, the fringes remain almost equal-referring to the contrast-without the contrast inversion inherent to the classical self-imaging phenomenon. This effect is better observed in figure 7(b). In addition, the effect of the sinc function is also observed in figure 7(b), presenting areas with high and low modulation of the fringe intensity.

Finally, figure 7(c) shows the dependence of the intensity along the optical axis, considering the punctual polychromatic source in terms of the amount of polychromaticity. As one can see, by increasing the amount of polychromaticity, the intensity of the central fringe stabilizes to a constant value. In this case, this value is due to the stabilization of the fringes without contrast inversion. This fact is of interest for optical encoder technology, since it would allow placement of the reading head at any distance from the grating without losing or inverting contrast.

\section{Q3 References}

[1] Loewen E G and Popov E 1997 Diffraction Gratings and Applications (New York: Marcel Dekker)

[2] Palmer C 2000 Diffraction Grating Handbook (New York: Richardson Grating Laboratory)

[3] Patorski K 1989 The self-imaging phenomenon and its applications Prog. Opt. 27 1-108

[4] Lohmann A W 1971 An interferometer based on the Talbot effect Opt. Commun. 2 413-5
[5] Oreb B F and Dorsch R G 1994 Profilometry by phase-shifted Talbot images Appl. Opt. 33 7955-62

[6] Talbot W H F 1836 Facts relating to optical science Phil. Mag. 9 401-7

[7] Sugiyama Y, Matsui Y, Toyoda H, Mukozaka N, Ihori A, Abe T, Tabake M and Mizuno S 2008 A $3.2 \mathrm{kHz}, 14-\mathrm{Bit}$ optical absolute rotary encoder with a CMOS profile sensor IEEE Sensors J. 8 1430-6

[8] Zhang J H and Cai L 1998 Autofocus laser rotary encoder Appl. Opt. 37 2691-5

[9] Alonso J and Bernabeu E 1993 Use of effective focal lengths to describe laser-beam evolution after diffraction in radial gratings J. Opt. Soc. Am. A 10 1963-70

[10] Koch J A, Cui S and McNeill M A 1999 Effective-focal-length calculations and measurements for a radial diffraction grating J. Opt. Soc. Am. A 16 2690-4

[11] Salgado-Remacha F J, Torcal-Milla F J, Sanchez-Brea L M and Bernabeu E 2011 Use of steel substrates in diffractive optics: near field oh high surface quality steel tape gratings $O p t$. Lasers Eng. 49 356-60

[12] Lutey A H A 2013 An improved model for nanosecond pulsed laser ablation of metals J. Appl. Phys. 114083108

[13] Hsieh C T and Lee C K 1999 Cylindrical-type nanometerresolution laser diffractive optical encoder Appl. Opt. 38 4743-50

[14] Tonchev S, Jourlin Y, Veillas C, Reynaud S, Lyndin N, Parriaux O, Laukkanen J and Kuittinen M 2012 Subwavelength cylindrical grating by holistic phase-mask coordinate transform Opt. Express 20 7946-53

[15] Parriaux O, Jourlin Y and Lyndin N 2010 Cylindrical Grating Rotation Sensor Patent Number US20100245841 A1

[16] Torcal-Milla F J, Sanchez-Brea L M and Bernabeu E 2007 Talbot effect with rough reflection gratings Appl. Opt. 46 3668-73

[17] Sanchez-Brea L M, Torcal-Milla F J and Bernabeu E 2007 Talbot effect in metallic gratings under Gaussian illumination Opt. Commun. 278 23-7

[18] Garcia-Rodriguez L, Alonso J and Bernabeu E 2004 Grating pseudo-imaging with polychromatic and finite extension sources Opt. Express 12 2529-41

[19] Sanchez-Brea L M, Saez Landete J, Alonso J and Bernabeu E 2008 Invariant grating pseudoimaging using polychromatic light and a finite extension source Appl. Opt. 47 1470-7

[20] Beutler H G 1945 The theory of the concave grating J. Opt. Soc. Am. 35 311-49

[21] Haber H 1950 The torus grating J. Opt. Soc. Am. 40 153-65

[22] Prieto-Blanco X, Montero-Orille C, Gonzalez-Núñez H, Mouriz M D, López-Lago E and de la Fuente R 2009 Imaging with classical spherical diffraction gratings: the quadrature configuration J. Opt. Soc. Am. A 26 2400-9

[23] Woodgate B E 1974 Cylindrical and spherical gratings J. Opt. Soc. Am. 64 654-61

[24] Torcal-Milla F J, Sanchez-Brea L M, Salgado-Remacha F J and Bernabeu E 2010 Self-imaging of curved gratings Opt. Commun. 283 3869-73 


\section{QUERY FORM}

JOURNAL: Journal of Optics

Author: F J Torcal-Milla et al

TITLE: Near field diffraction of cylindrical convex gratings

ARTICLE ID: jopt507941

The layout of this article has not yet been finalized. Therefore this proof may contain columns that are not fully balanced/ matched or overlapping text in inline equations; these issues will be resolved once the final corrections have been incorporated.

\section{SQ1}

Please be aware that the colour figures in this article will only appear in colour in the online version. If you require colour in the printed journal and have not previously arranged it, please contact the Production Editor now.

\section{Page}

Q1

We have been provided funding information for this article as below. Please confirm whether this information is correct. Ministry of Science and Innovation, Spain: DPI2011-27851.

\section{Page 2}

Q2

Please confirm whether the word 'one' can be replaced with 'point'.

\section{Page 8}

Q3

Please check the details for any journal references that do not have a link as they may contain some incorrect information. 